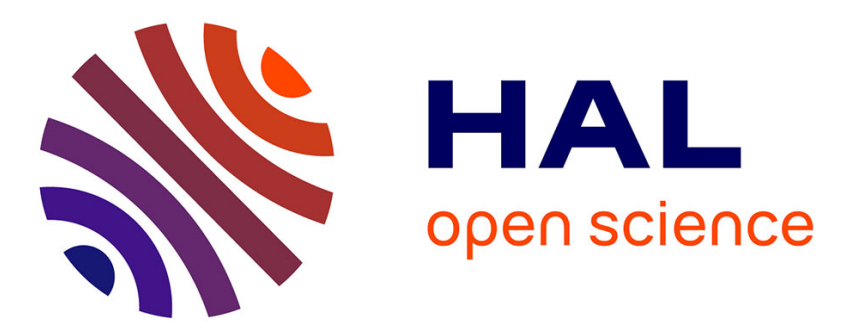

\title{
Finite-time guaranteed state estimation for discrete-time systems with disturbances
}

\author{
Thach Ngoc Dinh, Frédéric Mazenc, Tarek Raïssi
}

\section{To cite this version:}

Thach Ngoc Dinh, Frédéric Mazenc, Tarek Raïssi. Finite-time guaranteed state estimation for discretetime systems with disturbances. SysTol 2019 - 4th International Conference on Control and FaultTolerant Systems, Sep 2019, Casablanca, Morocco. pp.342-347, 10.1109/SYSTOL.2019.8864742 . hal-02342708

\section{HAL Id: hal-02342708 \\ https://hal.inria.fr/hal-02342708}

Submitted on 1 Nov 2019

HAL is a multi-disciplinary open access archive for the deposit and dissemination of scientific research documents, whether they are published or not. The documents may come from teaching and research institutions in France or abroad, or from public or private research centers.
L'archive ouverte pluridisciplinaire HAL, est destinée au dépôt et à la diffusion de documents scientifiques de niveau recherche, publiés ou non, émanant des établissements d'enseignement et de recherche français ou étrangers, des laboratoires publics ou privés. 


\title{
Finite-time guaranteed state estimation for discrete-time systems with disturbances
}

\author{
Thach Ngoc Dinh ${ }^{1}$, Frederic Mazenc ${ }^{2}$ and Tarek Raïssi ${ }^{1}$
}

\begin{abstract}
In this paper, we consider a family of discrete-time systems with input and output. A new technique, based on past values of the output which have been employed to construct some finite-time observers for continuous-time systems in the literature, is proposed for discrete-time case. The systems are affected by additive disturbances and disturbances in the output. Exact estimation or approximate estimation can be achieved depending on the absence or the presence of unknown but bounded uncertainties, respectively.
\end{abstract}

\section{INTRODUCTION}

The problem of designing state observers for dynamical systems, including automatic control systems, is a classical one and has rich history. For the purpose of feedback control to a system such as vehicles, chemical plants and biological systems, all of its state space variables must be available. However in general in engineering, some of the state variables are not available for measurements or measure of them is problematic (e.g. it is too expensive to measure all state variables). Thus, control designers have to face with the problem of estimating system state space variables based on a model and a limited set of possible input/output measurements. This can be done by building a dynamic extension, called an observer or an estimator. Roughly speaking, an observer is an auxiliary dynamic system coupled to the original system through the measured inputs and outputs. In many contributions, designs of observers are proposed. We can mention here traditional state estimators which are very popular, such as Luenberger observer [14]. Another cutting-edge technique of guaranteed state estimation is the interval observer based technique which was introduced two decades ago in [9]. Typically, interval observers give accurate component-wise estimations at any time instant when upper and lower bounds of the initial state are known, see for instance [20], [15], [16] and the references therein. The usefulness of interval estimates is evident not only for feedback control purpose but also for monitoring purposes when large disturbances or uncertainties are present. Therefore, the topic is interesting from the mathematical point of view, and also has an important potential for use in a lot of industrial applications [1], [6]. A promising concept, called fixed-time stability, is proposed in [19]. Using this concept, fixed-time controllers were proposed to ensure that

\footnotetext{
${ }^{1}$ Thach Ngoc Dinh and Tarek Raïssi are with Conservatoire National des Arts et Métiers (CNAM), Cedric-Lab, 292 rue St-Martin, 75141 Paris Cedex 03, FRANCE ngoc-thach.dinh@lecnam.net, tarek.raissiecnam.fr

${ }^{2}$ Frederic Mazenc is with EPI DISCO Inria-Saclay, L2S, CNRS CentraleSupélec, 3 rue Joliot Curie, 91192 Gif-sur-Yvette, FRANCE frederic.mazencel2s.centralesupelec.fr
}

some control performances are achieved regardless of the initial conditions of the system [7]. Uniform robust exact differentiators were proposed in [2], [5], [13], [21] based on a Lyapunov analysis or homogeneity properties. A fixedtime observer, with linear matrix inequality algorithms for tuning the observer parameters, was introduced in [12] for linear systems. Based on uniform robust exact differentiators, a uniformly convergent sliding mode observer was proposed in [18]. Although the settling time estimate does not depend on the initial conditions of the system in many works, it cannot be easily tuned and it is very over-estimated.

In the present work, a new approach of finite-time guaranteed state estimations for estimating state variables is proposed where no appropriate knowledge of the initial conditions is known and the systems have no monotonicity property. The aim of this note is different from interval observers presented above, which always request an appropriate knowledge in terms of an upper and lower bound of the initial conditions and a direct or indirect notion of nonnegative and cooperative system. The approach is based on formulas incorporating past values of the input and the output of the studied plant. The technique has been developed in several contexts: in particular, some works are devoted to families of linear systems [8], and others are devoted to nonlinear systems [22], [11], [17]. A common feature of these results is that they apply only to continuoustime systems. On the other hand, discrete-time systems are very important from a theoretical as well as an applied point of view and the problem of constructing observers or dynamic output feedbacks for them has been extensively studied [10], [4], [23]. Furthermore, it is worth noting that discretization techniques transform continuous-time systems into discrete-time systems and systems with sampled data often lead to discrete-time system [3]. These systems are usually affected by two types of bounded deterministic timevarying disturbances: in the dynamics and in the output. This fact motivates our work. Employing past values of the input and the output only, two goals can be achieved. In the absence of unknown uncertainties and after a finite time, the exact values of the solutions are given. Next when unknown disturbances are present and are upper and lower bounded by known constant vectors, after a finite time, the formulas we exhibit provide upper and lower bounds for each component of the solutions, as interval observers do.

The paper is organized as follows. Setups and objectives are introduced in Section II. Exact values of the solutions in the absence of uncertainties are given in Section III. Intervals for the solutions in the presence of uncertainties are 
constructed in Section IV. The main results are illustrated in Section V. Concluding remarks are given in Section VI.

Notations, definitions. The set of natural numbers and real numbers are denoted by $\mathbb{N}$ and $\mathbb{R}$, respectively. The Euclidean norm of a vector $x \in \mathbb{R}^{n}$ is denoted by $|x|$. Inequalities must be understood component-wise, i.e., for $x_{a}=\left[x_{a, 1}, \ldots, x_{a, n}\right]^{\top} \in \mathbb{R}^{n}$ and $x_{b}=\left[x_{b, 1}, \ldots, x_{b, n}\right]^{\top} \in \mathbb{R}^{n}$, $x_{a} \leq x_{b}$ if and only if, for all $i \in\{1, \ldots, n\}, x_{a, i} \leq x_{b, i}$. For a square matrix $Q \in \mathbb{R}^{n \times n}$, the determinant of $Q$ is denoted $\operatorname{det}(Q)$ and we define $Q^{+}, Q^{-} \in \mathbb{R}^{n \times n}$ by $Q^{+}=\max (Q, 0)$ and $Q^{-}=Q^{+}-Q$.

\section{Setups AND OBJectives}

Consider the following discrete-time system:

$\left\{\begin{array}{l}x(k+1)=A x(k)+F(u(k), y(k))+d(k) \\ y(k)=C x(k)+v(k)\end{array}, \quad k \in \mathbb{N}\right.$.

where $x(k) \in \mathbb{R}^{n}$ is the state, $F$ is a nonlinear function, $C \in \mathbb{R}^{q \times n}, y(k) \in \mathbb{R}^{q}$ is the output, $u(k) \in \mathbb{R}^{p}$ is an input, and $d: \mathbb{N} \rightarrow \mathbb{R}^{n}, v: \mathbb{N} \rightarrow \mathbb{R}^{q}$ are respectively disturbances in the dynamics and in the output.

We introduce the following assumptions :

Assumption 1: The pair $(A, C)$ is observable and $A$ is invertible.

Assumption 2: There are known constant vectors $\underline{d} \in$ $\mathbb{R}^{n}, \bar{d} \in \mathbb{R}^{n}$ and $\underline{v} \in \mathbb{R}^{q}, \bar{v} \in \mathbb{R}^{q}$ such that for all $k \in \mathbb{N}$, the inequalities

$$
\begin{aligned}
& \underline{d} \leq d(k) \leq \bar{d} \\
& \underline{v} \leq v(k) \leq \bar{v}
\end{aligned}
$$

are satisfied.

Inspired by [17], the observer designs proposed in this note achieve two objectives simultaneously:

- An exact estimation for the solutions in finite time when the functions $d$ and $v$ are known.

- Design of two bounds for the solutions in finite time when the functions $d$ and $v$ are unknown but bounded by known values.

\section{Discussion of the Assumptions:}

- Assumption 1 implies that there is a matrix $L \in \mathbb{R}^{n \times q}$ such that the matrix

$$
H=A+L C \in \mathbb{R}^{n \times n}
$$

admits a spectral radius smaller than the modulus of any eigenvalue of $A$. Then, we prove in Lemma 1 in Appendix that in this case, there is an integer $h>0$ such that the matrix $H^{-h}-A^{-h}$ is invertible.

- The assumption that $A$ is invertible is not restictive at all because when $(A, C)$ is observable and $A$ is not invertible, we can always decompose $A x+F(u, y)$ in an alternative way so that the new matrix $A$ is invertible.

- Assumption 2 is realistic and is frequently satisfied in practice. It can be relaxed by allowing the bounds to depend on time $k$ but for the sake of the simplicity, we restrict ourselves to the case where they are constant.

\section{EXACT ESTIMATION}

The results of this section provide with exact estimations of the solutions in finite time, but they can be applied only when the functions $d$ and $v$ are known. Let us state and prove the following result:

Theorem 1: Let the system (1) satisfy Assumptions 1. Let $L \in \mathbb{R}^{n \times q}$ and $h \in \mathbb{N}, h \geq 1$ be such that the matrix $H^{-h}-A^{-h}$ is invertible. Then, for a given input $u(k)$, any solution $x(k)$ of the system (1) which exists over $\mathbb{N}$ satisfies, for all $k \geq h$,

$$
\begin{aligned}
x(k)= & -E_{h} \sum_{\ell=k-h}^{k-1} A^{k-h-\ell-1} F(u(\ell), y(\ell)) \\
& +E_{h} \sum_{\ell=k-h}^{k-1} H^{k-h-\ell-1}[F(u(\ell), y(\ell))-L y(\ell)] \\
& -E_{h} \sum_{\ell=k-h}^{k-1} A^{k-h-\ell-1} d(\ell) \\
& +E_{h} \sum_{\ell=k-h}^{k-1} H^{k-h-\ell-1} L v(\ell)
\end{aligned}
$$

with

$$
E_{h}=\left(H^{-h}-A^{-h}\right)^{-1}
$$

Proof: From the definition of the output $y$ and the definition of $H$, we deduce that the system (1) admits two representations:

$$
\begin{aligned}
x(k+1)= & A x(k)+F(u(k), y(k))+d(k), \\
x(k+1)= & H x(k)+F(u(k), y(k)) \\
& -L y(k)+d(k)+L v(k) .
\end{aligned}
$$

By combining all equations of these two systems between two values $m_{1} \in \mathbb{N}, m_{2} \in \mathbb{N}$ and $m_{1} \geq m_{2}$, we obtain the equalities

$$
\begin{array}{r}
x\left(m_{1}\right)=A^{m_{1}-m_{2}} x\left(m_{2}\right)+\sum_{\ell=m_{2}}^{m_{1}-1} A^{m_{1}-\ell-1}[F(u(\ell), y(\ell)) \\
+d(\ell)], \quad(9) \\
x\left(m_{1}\right)=H^{m_{1}-m_{2}} x\left(m_{2}\right)+\sum_{\ell=m_{2}}^{m_{1}-1} H^{m_{1}-\ell-1}[F(u(\ell), y(\ell)) \\
-L y(\ell)+d(\ell)+L v(\ell)] .
\end{array}
$$

Now, consider a value $k \geq h$. Then selecting $m_{2}=k-h$ and $m_{1}=k$, the equalities (9)-(10) give

$$
\begin{gathered}
x(k)=A^{h} x(k-h)+\sum_{\ell=k-h}^{k-1} A^{k-\ell-1}[F(u(\ell), y(\ell))+d(\ell)], \\
x(k)=H^{h} x(k-h)+\sum_{\ell=k-h}^{k-1} H^{k-\ell-1}[F(u(\ell), y(\ell)) \\
-L y(\ell)+d(\ell)+L v(\ell)] .
\end{gathered}
$$


As an immediate consequence, we have

$$
\begin{aligned}
& \left(H^{-h}-A^{-h}\right) x(k) \\
& =-\sum_{\ell=k-h}^{k-1} A^{k-h-\ell-1}[F(u(\ell), y(\ell))+d(\ell)] \\
& +\sum_{\ell=k-h}^{k-1} H^{k-h-\ell-1}[F(u(\ell), y(\ell))-L y(\ell)+d(\ell)+L v(\ell)] .
\end{aligned}
$$

Since $H^{-h}-A^{-h}$ is invertible, we deduce that (5) is satisfied.

The formula (5) may contain many terms because $h$ may be large and thus many values have to be stored. To overcome this drawback, we propose an alternative solution which is based on a dynamic extensions.

Theorem 2: Let the system (1) satisfy the Assumptions 1 , let $L$ and $h$ be defined as in Theorem 1 and let $u$ be a given input. Consider the dynamic extensions

$$
\hat{x}(k+1)=A \hat{x}(k)+F(u(k), y(k))+d(k)
$$

and

$$
\begin{aligned}
x_{*}(k+1)= & H x_{*}(k)+F(u(k), y(k)) \\
& -L y(k)+d(k)+L v(k) .
\end{aligned}
$$

Consider a solution $x(k)$ of (1) defined over $\mathbb{N}$. Then, for all $k \geq h$,

$$
\begin{array}{r}
x(k)=E_{h}\left[H^{-h} x_{*}(k)-x_{*}(k-h)\right. \\
\left.-A^{-h} \hat{x}(k)+\hat{x}(k-h)\right] .
\end{array}
$$

Remark 1: Notice that (14) is a classical observer for the system (1) when disturbances are known.

Proof: Consider a solution $\left(\hat{x}(k), x_{*}(k)\right)$ of (13)-(14) associated with the solution $x(k)$ defined over $\mathbb{N}$. Then, arguing as we did in the proof of Theorem 1, we have

$$
\begin{aligned}
\hat{x}\left(m_{1}\right)= & A^{m_{1}-m_{2}} \hat{x}\left(m_{2}\right)+\sum_{\ell=m_{2}}^{m_{1}-1} A^{m_{1}-\ell-1}[F(u(\ell), y(\ell)) \\
x_{*}\left(m_{1}\right)= & H^{m_{1}-m_{2}} x_{*}\left(m_{2}\right)+\sum_{\ell=m_{2}}^{m_{1}-1} H^{m_{1}-\ell-1} \times \\
& \times[F(u(\ell), y(\ell))-L y(\ell)+d(\ell)+L v(\ell)] . \quad(17)
\end{aligned}
$$

It follows that for all $k \geq h$,

$$
\begin{aligned}
& \sum_{\ell=k-h}^{k-1} A^{k-h-\ell-1}[F(u(\ell), y(\ell))+d(\ell)] \\
& =A^{-h} \hat{x}(k)-\hat{x}(k-h), \\
& \sum_{\ell=k-h}^{k-1} H^{k-h-\ell-1}[F(u(\ell), y(\ell))-L y(\ell)+d(\ell)+L v(\ell)] \\
& =H^{-h} x_{*}(k)-x_{*}(k-h) .
\end{aligned}
$$

Combining (5), (18) and (19), we obtain, for all $k \geq h$,

$$
\begin{aligned}
E_{h}^{-1} x(k)= & H^{-h} x_{*}(k)-x_{*}(k-h) \\
& -A^{-h} \hat{x}(k)+\hat{x}(k-h) .
\end{aligned}
$$

This allows us to conclude.

\section{APPROXIMATE ESTIMATION}

Theorems 1 and 2 give in finite time the exact value of any solution $x(k)$ of (1). However, these estimations cannot be used when the disturbances $d$ and $v$ are unknown. The second objective of the present note is to overcome this limitation by assuming only that the bounds $\underline{d}, \bar{d}$ of $d$ and $\underline{v}, \bar{v}$ of $v$ are known.

In this section, we consider the case where Assumptions 1 and 2 are satisfied and the matrix $L$ is selected as described in (4). Next, we define the following matrices

$$
F_{h}=-E_{h} A^{-(h+1)}, \quad G_{h}=E_{h} H^{-(h+1)} .
$$

and the vectors

$$
\begin{aligned}
& d_{L}=\left(F_{h} \sum_{\ell=1}^{h} A^{\ell}\right)^{+} \bar{d}-\left(F_{h} \sum_{\ell=1}^{h} A^{\ell}\right)^{-} \underline{d} \\
& d_{S}=\left(F_{h} \sum_{\ell=1}^{h} A^{\ell}\right)^{+} \underline{d}-\left(F_{h} \sum_{\ell=1}^{h} A^{\ell}\right)^{-} \bar{d} \\
& v_{L}=\left(G_{h} \sum_{\ell=1}^{h} H^{\ell} L\right)^{+} \bar{v}-\left(G_{h} \sum_{\ell=1}^{h} H^{\ell} L\right)^{-} \underline{v} \\
& v_{S}=\left(G_{h} \sum_{\ell=1}^{h} H^{\ell} L\right)^{+} \underline{v}-\left(G_{h} \sum_{\ell=1}^{h} H^{\ell} L\right)^{-} \bar{v} .
\end{aligned}
$$

We are ready to state and prove the following result:

Theorem 3: Let the system (1) satisfy Assumptions 1 and 2 and let $L$ and $h \in \mathbb{N}, h \geq 1$ be such that $E_{h}$ given in (6) is well-defined. Let $u$ be a given input and consider a solution of the system (1) defined over $\mathbb{N}$. Then, for all integer $k \geq h$, the inequalities

$$
\underline{x}(k) \leq x(k) \leq \bar{x}(k)
$$

with

$$
\begin{aligned}
\bar{x}(k)= & -E_{h} \sum_{\ell=k-h}^{k-1} A^{k-h-\ell-1} F(u(\ell), y(\ell)) \\
& +E_{h} \sum_{\ell=k-h}^{k-1} H^{k-h-\ell-1}[F(u(\ell), y(\ell))-L y(\ell)] \\
& +d_{L}+v_{L}, \\
\underline{x}(k)= & -E_{h} \sum_{\ell=k-h}^{k-1} A^{k-h-\ell-1} F(u(\ell), y(\ell)) \\
& +E_{h} \sum_{\ell=k-h}^{k-1} H^{k-h-\ell-1}[F(u(\ell), y(\ell))-L y(\ell)] \\
& +d_{S}+v_{S},
\end{aligned}
$$

hold. 
Proof: From (5), we have

$$
\begin{aligned}
x(k)= & -E_{h} \sum_{\ell=k-h}^{k-1} A^{k-h-\ell-1} F(u(\ell), y(\ell)) \\
& +E_{h} \sum_{\ell=k-h}^{k-1} H^{k-h-\ell-1}[F(u(\ell), y(\ell))-L y(\ell)] \\
& -E_{h} A^{-(h+1)} \sum_{\ell=k-h}^{k-1} A^{k-\ell} d(\ell) \\
& +E_{h} H^{-(h+1)} \sum_{\ell=k-h}^{k-1} H^{k-\ell} L v(\ell) .
\end{aligned}
$$

From (29) and the definition of $F_{h}$ and $G_{h}$ in (21), it follows that, for all $k \geq h$,

$$
\begin{aligned}
x(k)= & -E_{h} \sum_{\ell=k-h}^{k-1} A^{k-h-\ell-1} F(u(\ell), y(\ell)) \\
& +E_{h} \sum_{\ell=k-h}^{k-1} H^{k-h-\ell-1}[F(u(\ell), y(\ell))-L y(\ell)] \\
& +F_{h} \sum_{\ell=1}^{h} A^{\ell} d(k-\ell)+G_{h} \sum_{\ell=1}^{h} H^{\ell} L v(k-\ell) .
\end{aligned}
$$

From Assumption 2, we deduce that, for all $k \geq h$,

$$
\begin{aligned}
& \left(F_{h} \sum_{\ell=1}^{h} A^{\ell}\right)^{+} \underline{d}-\left(F_{h} \sum_{\ell=1}^{h} A^{\ell}\right)^{-} \bar{d} \\
& \leq F_{h} \sum_{\ell=1}^{h} A^{\ell} d(k-\ell) \\
& \leq\left(F_{h} \sum_{\ell=1}^{h} A^{\ell}\right)^{+} \bar{d}-\left(F_{h} \sum_{\ell=1}^{h} A^{\ell}\right)^{-} \underline{d} \\
& \left(G_{h} \sum_{\ell=1}^{h} H^{\ell} L\right)^{+} \underline{v}-\left(G_{h} \sum_{\ell=1}^{h} H^{\ell} L\right)^{-} \bar{v} \\
& \leq G_{h} \sum_{\ell=1}^{h} H^{\ell} L v(k-\ell) \\
& \leq\left(G_{h} \sum_{\ell=1}^{h} H^{\ell} L\right)^{+} \bar{v}-\left(G_{h} \sum_{\ell=1}^{h} H^{\ell} L\right)^{-} \underline{v} .
\end{aligned}
$$

It follows that

$$
\begin{gathered}
d_{S} \leq F_{h} \sum_{\ell=1}^{h} A^{\ell} d(k-\ell) \leq d_{L}, \\
v_{S} \leq G_{h} \sum_{\ell=1}^{h} H^{\ell} L v(k-\ell) \leq v_{L},
\end{gathered}
$$

with

$$
\begin{aligned}
& d_{L}=\left(F_{h} \sum_{\ell=1}^{h} A^{\ell}\right)^{+} \bar{d}-\left(F_{h} \sum_{\ell=1}^{h} A^{\ell}\right)^{-} \underline{d}, \\
& d_{S}=\left(F_{h} \sum_{\ell=1}^{h} A^{\ell}\right)^{+} \underline{d}-\left(F_{h} \sum_{\ell=1}^{h} A^{\ell}\right)^{-} \bar{d},
\end{aligned}
$$

and

$$
\begin{aligned}
& v_{L}=\left(G_{h} \sum_{\ell=1}^{h} H^{\ell} L\right)^{+} \bar{v}-\left(G_{h} \sum_{\ell=1}^{h} H^{\ell} L\right)^{-} \underline{v}, \\
& v_{S}=\left(G_{h} \sum_{\ell=1}^{h} H^{\ell} L\right)^{+} \underline{v}-\left(G_{h} \sum_{\ell=1}^{h} H^{\ell} L\right)^{-} \bar{v} .
\end{aligned}
$$

From (30), (31) and (32), we can conclude.

The motivations of Theorem 2 also motivate the following result:

Theorem 4: Let the system (1) satisfy the conditions in Theorem 3. Let $u(k)$ be a given input and consider a solution of (1) defined over $\mathbb{N}$. Let us introduce several dynamic extensions:

$$
\begin{aligned}
& z_{a}(k+1)=A z_{a}(k)+F(u(k), y(k)), \\
& z_{h}(k+1)=H z_{h}(k)+F(u(k), y(k))-L y(k),
\end{aligned}
$$

Then, for all $k \geq h$, the inequalities

$$
\underline{\Upsilon}\left(Z_{k}\right) \leq x(k) \leq \bar{\Upsilon}\left(Z_{k}\right),
$$

with $Z=\left(z_{a}, z_{h}\right)$ and the bounds $\bar{\Upsilon}, \underline{\Upsilon}$ are an estimated interval for the system (1) given by

$$
\begin{aligned}
& \bar{\Upsilon}\left(Z_{k}\right)=E_{h}\left[z_{a}(k-h)-A^{-h} z_{a}(k)\right. \\
& \left.+H^{-h} z_{h}(k)-z_{h}(k-h)\right]+d_{L}+v_{L}, \\
& \underline{\Upsilon}\left(Z_{k}\right)=E_{h}\left[z_{a}(k-h)-A^{-h} z_{a}(k)\right. \\
& \left.+H^{-h} z_{h}(k)-z_{h}(k-h)\right]+d_{S}+v_{S},
\end{aligned}
$$

where $d_{L}, d_{S}, v_{L}, v_{S}$ are the vectors defined in (22), (23), (24), (25), are satisfied.

Proof: For a solution $x(k)$ of (1) defined over $\mathbb{N}$, we have

$$
\begin{array}{r}
z_{a}(k)=A^{h} z_{a}(k-h)+\sum_{\ell=k-h}^{k-1} A^{k-\ell-1} F(u(\ell), y(\ell)), \\
z_{h}(k)=H^{h} z_{h}(k-h)+\sum_{\ell=k-h}^{k-1} H^{k-\ell-1}[F(u(\ell), y(\ell)) \\
-L y(\ell)] .
\end{array}
$$

These equalities rewrite as

$$
\begin{aligned}
-\sum_{\ell=k-h}^{k-1} A^{k-h-\ell-1} F(u(\ell), y(\ell)) & =z_{a}(k-h)-A^{-h} z_{a}(k), \\
\sum_{\ell=k-h}^{k-1} H^{k-h-\ell-1}[F(u(\ell), y(\ell)) & -L y(\ell)] \\
& =H^{-h} z_{h}(k)-z_{h}(k-h) .
\end{aligned}
$$


Theorem 3 ensures that the inequalites (26) hold. Then from these inequalities and the equalities (42) and (43), we deduce that the inequalities (39) are satisfied.

\section{ILLUSTRATIVE EXAMPLE}

In this section, consider the system

$$
\begin{aligned}
x_{1}(k+1) & =\frac{5}{4} x_{1}(k)+x_{2}(k)+\frac{1}{4} u_{1}(k)+\frac{1}{9} \sin (k), \\
x_{2}(k+1) & =-\frac{3}{8} x_{1}(k)+\frac{1}{8} u_{2}(k)+\frac{1}{9} \sin (k), \\
y(k) & =x_{1}(k)+\frac{1}{9} \sin \left(k^{2}\right) .
\end{aligned}
$$

System (46) is of the form (1) with $C=\left[\begin{array}{ll}1 & 0\end{array}\right]$,

$$
\begin{array}{r}
A=\left[\begin{array}{rr}
\frac{5}{4} & 1 \\
-\frac{3}{8} & 0
\end{array}\right], \quad F(u(k), y(k))=\left[\begin{array}{c}
\frac{1}{4} u_{1}(k) \\
\frac{1}{8} u_{2}(k)
\end{array}\right], \\
d(k)=\left[\begin{array}{c}
\frac{1}{9} \sin (k) \\
\frac{1}{9} \sin (k)
\end{array}\right] \text { and } v(k)=\frac{1}{9} \sin \left(k^{2}\right) .
\end{array}
$$

Notice that the pair $(A, C)$ is observable. The choice $L=$ $\left[\begin{array}{ll}-\frac{7}{8} & \frac{11}{32}\end{array}\right]^{\top}$ gives $H=A+L C=\left[\begin{array}{rr}\frac{3}{8} & 1 \\ -\frac{1}{32} & 0\end{array}\right]$.

Now to determine an analytical expression of the matrix $H^{-h}-A^{-h}$, let us observe that $R_{1} A R_{1}^{-1}=S_{1}$ with

$$
R_{1}=\left[\begin{array}{cc}
1 & \frac{4}{3} \\
1 & 2
\end{array}\right] \quad \text { and } \quad S_{1}=\left[\begin{array}{cc}
\frac{3}{4} & 0 \\
0 & \frac{1}{2}
\end{array}\right] \text {. }
$$

and $S_{2}=R_{2} H R_{2}^{-1}$ with

$$
R_{2}=\left[\begin{array}{ll}
1 & 4 \\
1 & 8
\end{array}\right] \text { and } S_{2}=\left[\begin{array}{cc}
\frac{1}{4} & 0 \\
0 & \frac{1}{8}
\end{array}\right] .
$$

Next, for an integer $h>0$, we have

$$
\begin{aligned}
H^{-h}-A^{-h} & =\left(H^{h}\right)^{-1}-\left(A^{h}\right)^{-1} \\
& =R_{2}^{-1} S_{2}^{-h} R_{2}-R_{1}^{-1} S_{1}^{-h} R_{1} \\
& =\left[\begin{array}{ll}
\alpha_{11} & \alpha_{12} \\
\alpha_{21} & \alpha_{22}
\end{array}\right],
\end{aligned}
$$

with

$$
\begin{aligned}
& \alpha_{11}=2\left(\frac{1}{4}\right)^{-h}-\left(\frac{1}{8}\right)^{-h}-3\left(\frac{3}{4}\right)^{-h}+2\left(\frac{1}{2}\right)^{-h}, \\
& \alpha_{12}=8\left(\frac{1}{4}\right)^{-h}-8\left(\frac{1}{8}\right)^{-h}-4\left(\frac{3}{4}\right)^{-h}+4\left(\frac{1}{2}\right)^{-h}, \\
& \alpha_{21}=\frac{1}{4}\left(\frac{1}{8}\right)^{-h}-\frac{1}{4}\left(\frac{1}{4}\right)^{-h}+\frac{3}{2}\left(\frac{3}{4}\right)^{-h}-\frac{3}{2}\left(\frac{1}{2}\right)^{-h}, \\
& \alpha_{22}=2\left(\frac{1}{8}\right)^{-h}-\left(\frac{1}{4}\right)^{-h}+2\left(\frac{3}{4}\right)^{-h}-3\left(\frac{1}{2}\right)^{-h} .
\end{aligned}
$$

The matrix $H^{-h}-A^{-h}$ is invertible for all $h \geq 2$ because

$$
\begin{aligned}
\operatorname{det}\left(H^{-h}-A^{-h}\right)= & \left(\frac{1}{32}\right)^{-h}-6\left(\frac{1}{16}\right)^{-h}-6\left(\frac{3}{16}\right)^{-h} \\
& +5\left(\frac{1}{8}\right)^{-h}+5\left(\frac{3}{32}\right)^{-h}+\left(\frac{3}{8}\right)^{-h} \\
= & \left(\frac{8}{3}\right)^{h}\left(2^{h}-1\right) \times \\
& \times\left(5 \times 2^{h}-5 \times 3^{h}+6^{h}-1\right),
\end{aligned}
$$

which is strictly positive when $h \geq 2$.

Then for all $h \geq 2$,

$$
E_{h}=\left(H^{-h}-A^{-h}\right)^{-1}=\left[\begin{array}{ll}
\epsilon_{11} & \epsilon_{12} \\
\epsilon_{21} & \epsilon_{22}
\end{array}\right],
$$

with

$$
\begin{aligned}
& \epsilon_{11}=\frac{2\left(\frac{1}{8}\right)^{-h}-\left(\frac{1}{4}\right)^{-h}+2\left(\frac{3}{4}\right)^{-h}-3\left(\frac{1}{2}\right)^{-h}}{\left(\frac{8}{3}\right)^{h}\left(2^{h}-1\right)\left(5 \times 2^{h}-5 \times 3^{h}+6^{h}-1\right)}, \\
& \epsilon_{12}=\frac{-8\left(\frac{1}{4}\right)^{-h}+8\left(\frac{1}{8}\right)^{-h}+4\left(\frac{3}{4}\right)^{-h}-4\left(\frac{1}{2}\right)^{-h}}{\left(\frac{8}{3}\right)^{h}\left(2^{h}-1\right)\left(5 \times 2^{h}-5 \times 3^{h}+6^{h}-1\right)}, \\
& \epsilon_{21}=\frac{-\frac{1}{4}\left(\frac{1}{8}\right)^{-h}+\frac{1}{4}\left(\frac{1}{4}\right)^{-h}-\frac{3}{2}\left(\frac{3}{4}\right)^{-h}+\frac{3}{2}\left(\frac{1}{2}\right)^{-h}}{\left(\frac{8}{3}\right)^{h}\left(2^{h}-1\right)\left(5 \times 2^{h}-5 \times 3^{h}+6^{h}-1\right)}, \\
& \epsilon_{22}=\frac{2\left(\frac{1}{4}\right)^{-h}-\left(\frac{1}{8}\right)^{-h}-3\left(\frac{3}{4}\right)^{-h}+2\left(\frac{1}{2}\right)^{-h}}{\left(\frac{8}{3}\right)^{h}\left(2^{h}-1\right)\left(5 \times 2^{h}-5 \times 3^{h}+6^{h}-1\right)} .
\end{aligned}
$$

We apply Theorem 2 and select the initial values $x_{1}(0)=$ $2.3, x_{2}(0)=1$ and the input $u_{1}=1$ and $u_{2}=2$. Then, for different values of the delay $h=2,5,10$, we implement the exact estimation of the state $x$ given by (15). The simulation result is plotted in Figs. 1-2-3.

Finally, we implement the dynamic extensions $x_{a}$ and $x_{h}$ given by (37)-(38), and the upper and lower bounds given by (40)-(41). Figs. 4-5 illustrate two examples where $h=3$ and $h=4$ respectively with the same initial values and input. We choose the known bounds of disturbances $\bar{d}=\left[\frac{1}{9} \frac{1}{9}\right]^{\top}$, $\underline{d}=\left[-\frac{1}{9}-\frac{1}{9}\right]^{\top}, \bar{v}=\frac{1}{9}$ and $\underline{v}=-\frac{1}{9}$.
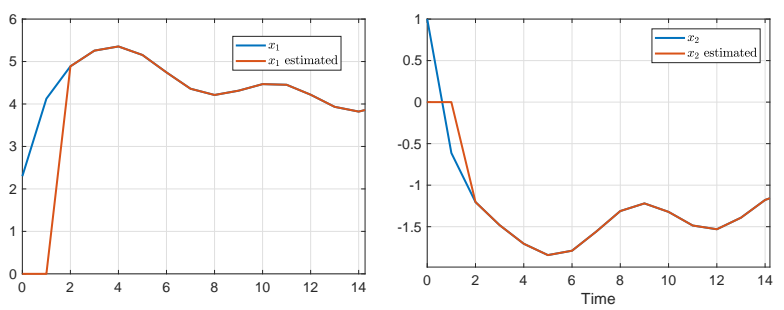

Fig. 1. Real state and exact estimation for $h=2$.
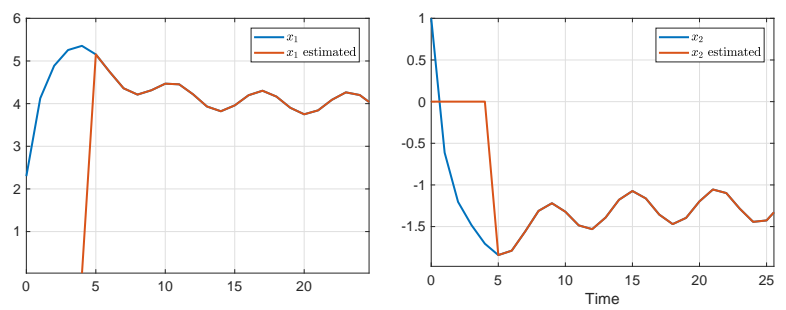

Fig. 2. Real state and exact estimation for $h=5$.

\section{CONCLUSION}

We have proposed a new technique of estimation of the solutions for a family of discrete-time nonlinear systems with disturbances in the dynamics and disturbances in the output. The key idea relies on the use of past values of the 

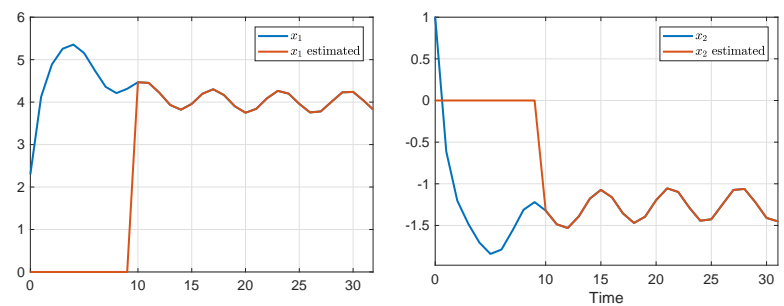

Fig. 3. Real state and exact estimation for $h=10$.
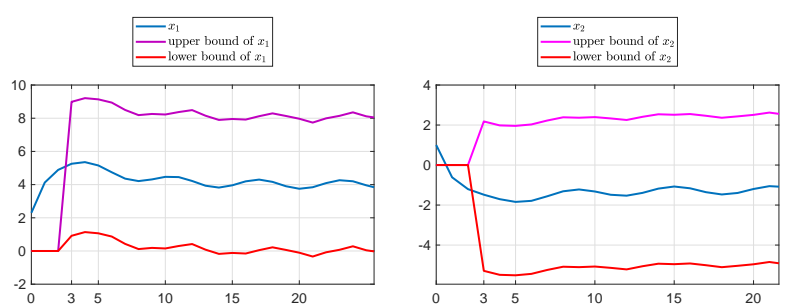

Fig. 4. Finite time interval estimation with upper and lower bounds for $h=3$.
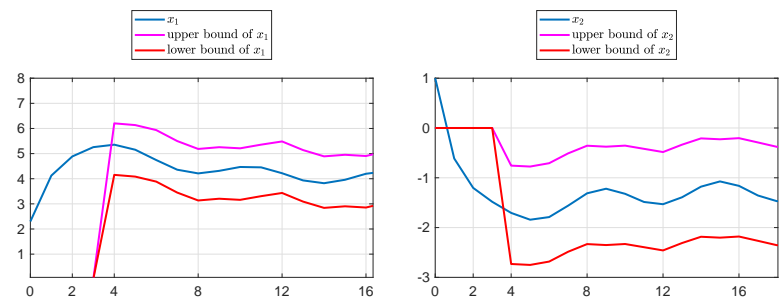

Fig. 5. Finite time interval estimation with upper and lower bounds for $h=4$.

input and the output of the studied system. No information on the bound of the initial conditions was needed in our development and we provided exact values of the solutions in the absence of disturbances and a lower and upper bounds when the disturbances are present after a finite time which can be tuned. Extension to more general families of nonlinear discrete-time systems and output feedback stabilization of systems can be considered for future works.

\section{APPENDIX}

Lemma 1: Let $(A, C)$ be an observable pair. Then there is a matrix $L$ such that the matrix $H=A+L C$ such that there is an integer $h_{*}$ such that for all $h \in \mathbb{N}, h \geq h_{*}$, the matrix $H^{-h}-A^{-h}$ is invertible.

Proof: Since the pair $(A, C)$ is observable, there is a matrix $L$ so that the spectral radius of $H$ is strictly less than 1 and smaller than the smallest norm of the eigenvalues of $A$. As an immediate consequence, $\lim _{r \rightarrow+\infty}\left|H^{r}\right|\left|A^{-r}\right|=0$. It follows that, there is an integer $h_{*}$ such that, for all $h \in \mathbb{N}$, $h \geq h_{*},\left|H^{h}\right|\left|A^{-h}\right|<1$. Consequently, it does not exist a vector $V \neq 0$ such that $\left(H^{h} A^{-h}-I\right) V=0$. Therefore $H^{h} A^{-h}-I$ is invertible. Note that the matrix $H^{-h}-A^{-h}$ is invertible if and only if $H^{h} A^{-h}-I$ is invertible. This concludes the proof.

\section{REFERENCES}

[1] V. Alcaraz-Gonzalez, V. Gonzalez-Alvarez, Robust Nonlinear Observers for Bioprocesses: Application to Wastewater Treatment, Lecture Notes in Control and Information Sciences, 361, pp. 119-164, 2007.

[2] M.T. Angulo, J.A. Moreno, L. Fridman, Robust exact uniformly convergent arbitrary order differentiator, Automatica, 49, pp. 24892495, 2013.

[3] K.J. Astrom, B. Wittenmark, Computer Controlled Systems. Theory and Design, (3rd edn). Printice-Hall: Englewood Cliffs, NJ, 1997.

[4] M. Boutayeb, M. Darouach. A reduced-order observer for nonlinear discrete-time systems. Systems \& Control Letters, 39, pp. 141?151, 2000

[5] E. Cruz-Zavala, J. A. Moreno, L. M. Fridman, Uniform robust exact differentiator. IEEE Transactions on Automatic Control, 56, pp. 2727$2733,2011$.

[6] S. Ifqir, N. A. Oufroukh, D. Ichalal, S. Mammar, Vehicle Lateral Dynamics Estimation using Switched Unknown Inputs Interval Observers: Experimental Validation. American Control Conference, Milwaukee, USA, pp. 1138-1143, 2018.

[7] M. Defoort, A. Polyakov, G. Demesure, M. Djemai, K. Veluvolu, Leader-follower fixed-time consensus for multiagent systems with unknown nonlinear inherent dynamics. IET Control Theory and Applications, 9(14), pp. 2165-2170, 2015.

[8] R. Engel, G. Kreisselmeier, A Continuous-Time Observer Which Converges in Finite Time. IEEE Transactions on Automatic Control, 47, pp. 1202-1204, 2002.

[9] J.L. Gouzé, A. Rapaport, Z. Hadj-Sadok, Interval observers for uncertain biological systems. Ecological Modelling 2000; 133:45?56.

[10] I. Karafyllis, C. Kravaris, On the observer problem for discrete-time control systems. IEEE Transactions on Automatic Control, 52, pp. 1225, 2007.

[11] J. Liu, S. Laghrouche, M. Wack, Finite time observer design for a class of nonlinear systems with unknown inputs. American Control Conference, Washington, USA, pp. 286-291, 2013.

[12] F. Lopez-Ramirez, A. Polyakov, D. Efimov, W. Perruquetti, Finite-time and fixed-time observers design via implicit Lyapunov function. 15th European Control Conference, Aalborg, Denmark, pp. 289-294, 2016.

[13] F. Lopez-Ramirez, A. Polyakov, D. Efimov, W. Perruquetti, Fixed-time output stabilization of a chain of integrators, 55th IEEE Conference on Decision and Control. Las Vegas, USA, pp. 3886-3891, 2016.

[14] D.G. Luenberger, An introduction to observers. IEEE Transactions on Automatic Control, 16, pp. 596-602, 1971.

[15] F. Mazenc, O. Bernard, Interval observers for linear time-invariant systems with disturbances. Automatica, 47, pp. 140-147, 2011.

[16] F. Mazenc, T.N. Dinh, Construction of Interval Observers for Continuous-time Systems with Discrete Measurements. Automatica, 50, pp. 2555-2560, 2014.

[17] F. Mazenc, E. Fridman, W. Djema, Estimation of solutions of observable nonliear systems with disturbances. Systems \& Control Letters, 79, pp. 47-58, 2015.

[18] D. Mincarelli, A. Pisano, T. Floquet, E. Usai, Uniformly convergent sliding mode-based observation for switched linear systems. International Journal of Robust and Nonlinear Control, 26, pp. 1549-1564, 2016

[19] A. Polyakov, Nonlinear feedback design for fixed-time stabilization of linear control systems. IEEE Transactions on Automatic Control, 57, pp. 2106-2110, 2012

[20] T. Raissi, D. Efimov, A. Zolghadri, Interval State Estimation for a Class of Nonlinear Systems. IEEE Transactions on Automatic Control, 57, pp. 260-265, 2012.

[21] H. Rios, A.R. Teel, A hybrid observer for fixed-time state estimation of linear systems. 55th IEEE Conference on Decision and Control, Las Vegas, USA, pp. 5408-5413, 2016.

[22] F. Sauvage, M. Guay, D. Dochain, Design of a Nonlinear Finite-Time Converging Observer for a Class of Nonlinear Systems. Journal of Control Science and Engineering, 2007.

[23] M. Xiao, N. Kazantzis, C. Kravaris, A.J. Krener, Nonlinear discretetime observer design with linearizable error dynamics. IEEE Transactions on Automatic Control, 48, 622-626, 2003. 